\title{
Apathy syndrome in a patient previously treated with selective serotonin reuptake inhibitors for depression
}

\author{
Hye-Geum Kim, Bon-Hoon Koo, Seung Woo Lee, Eun-Jin Cheon
}

Department of Psychiatry, Yeungnam University College of Medicine, Daegu, Korea

Received: January 25, 2019

Revised: March 5, 2019

Accepted: March 6, 2019

Corresponding author:

Eun-Jin Cheon

Department of Psychiatry,

Yeungnam University College of

Medicine, 170, Hyeonchung-ro,

Nam-gu, Daegu 42415, Korea

Tel: +82-53-620-3340

Fax: +82-53-629-0256

E-mail: pyejc@ynu.ac.kr
There is considerable overlap in the clinical presentations of apathy and depression. However, differential diagnosis between apathy and other psychiatric conditions, including depression and dementia, is important. In this report, we present the case of a 67-year-old woman with a history of receiving selective serotonin reuptake inhibitor (SSRI) treatment for depression. Differential diagnosis between treatment-resistant depression and SSRI-induced apathy syndrome was required. The symptoms of her apathy syndrome were relieved after the discontinuation of SSRIs and the addition of olanzapine, methylphenidate, and modafinil. Furthermore, we briefly review related literature in this article.

Keywords: Apathy; Depression; Serotonin reuptake inhibitor

\section{Introduction}

Apathy is a behavioral syndrome characterized by a decrease in interest, motivation, or initiation of action. Apathy may present in a similar manner as depression. However, Marin [1] emphasized the importance of differentiating between apathy and other psychiatric conditions, including depression, dementia, delirium, abulia, akinesia, despair, and demoralization. He proposed the following definition of apathy: a syndrome in which there is a primary absence of motivation that is not attributable to cognitive impairment, emotional distress, or diminished level of consciousness [1]. Many patients with apathy syndrome reported that "this feeling was unlike the lack of motivation they had sometimes experienced during prior episodes of depression," or that "their feelings of apathy bore no relationship to depression."

One reason for the importance of differentiating apathy syndrome from depression is that apathy syndrome has been reported in a number of patients receiving selective serotonin reuptake inhibitor (SSRI) treatment over the last decade [2]. Apathy also serves as a behavioral marker for rapidly progressing dementia, with a greater decline in cognitive, functional, and emotional impairment [3]. The presence of apathy is linked to deficits in the performance of activities of daily living and a functional decline [4]. Thus, the prompt recognition and treatment of apathy are crucial [4].

Here, we report the case of a patient who experienced apathy syndrome that was difficult to differentiate from depression and dementia. Written informed consent for the publication of this case report, approved by the institutional review board (YUMC 2017-12-022), was obtained from the patient.

\section{Case}

A 67-year-old woman with a remote history of depression was referred to our clinic due to the presentation of resistant depressive symptoms despite being treated with multiple antidepressant drugs, including escitalopram, paroxetine, duloxetine, and mirtazapine. She was admitted to the psychiatric clinic for approxi- 
mately a month with depression diagnosed about 2 years previously. Subsequently, her symptoms had improved. About 3 months previously, she relapsed and was admitted to the same clinic again, where she received medications including duloxetine $60 \mathrm{mg}$, mirtazapine $30 \mathrm{mg}$, escitalopram $20 \mathrm{mg}$, aripiprazole 1 $\mathrm{mg}$, and quetiapine $75 \mathrm{mg}$ for 2 months. However, her symptoms did not improve with the medication, and she was referred to our clinic. The depression symptoms included diminished interest, loss of energy, psychomotor agitation, weight loss, and insomnia. She reported that she was lethargic and did not want to do anything but she was not particularly depressed and had not experienced any increase in stress recently. She was admitted to our department of psychiatry for accurate assessment and treatment. The patient received a score of 26 on the Hamilton Depression Rating Scale. In addition to depressive symptoms, she had experienced cognitive impairment with gradual onset. She had a score of 22/30 on the Korean-Mini Mental State Examination, 1 on the Clinical Dementia Rating, and 4 on the Global Deterioration Scale. Her cognitive functions, including memory, executive function, and language function, were particularly impaired. In a structural brain imaging study, no degenerative changes other than a small aneurysm and internal carotid artery stenosis that had already been diagnosed were observed (Fig. 1). The results of the brain single-photon emission computed tomography for the evaluation of brain function indicated reduced perfusion in both anterior cingulate gyri (Fig. 2). She had no other medical history.

During the evaluation, we prescribed antidepressants such as fluoxetine $60 \mathrm{mg}$, venlafaxine $225 \mathrm{mg}$, mirtazapine $30 \mathrm{mg}$, and aripiprazole $5 \mathrm{mg}$, because the patient's symptoms for depression required immediate treatment. She continued to take the medications for more than a month, but the symptoms did not improve, and she appeared to be deteriorating. In a reassessment of the patient's medical history we focused on her apathy symptoms instead of her depression symptoms. At that point, the patient was evaluated and received a score of 72 on the Apathy Evaluation Scale (AES). This 18-item scale assesses apathy in behavioral, cognitive, and emotional domains over the previous 4 weeks. Scores range from 18 to 72 , with higher scores indicating greater apathy. Subsequently, we first reduced and then discontinued all antidepressants, including SSRIs. Olanzapine and sleeping pills were continued to control her insomnia and agitation. Subsequently, she became slightly more active but this change was not sufficient. We prescribed methylphenidate and gradually increased its dosage to $25 \mathrm{mg}$. We then added modafinil $200 \mathrm{mg}$ to her treatment regime. Following these changes of medication, her score on the AES improved to 35, and she continued performing her daily activities after discharge. The drugs she was taking at dis-

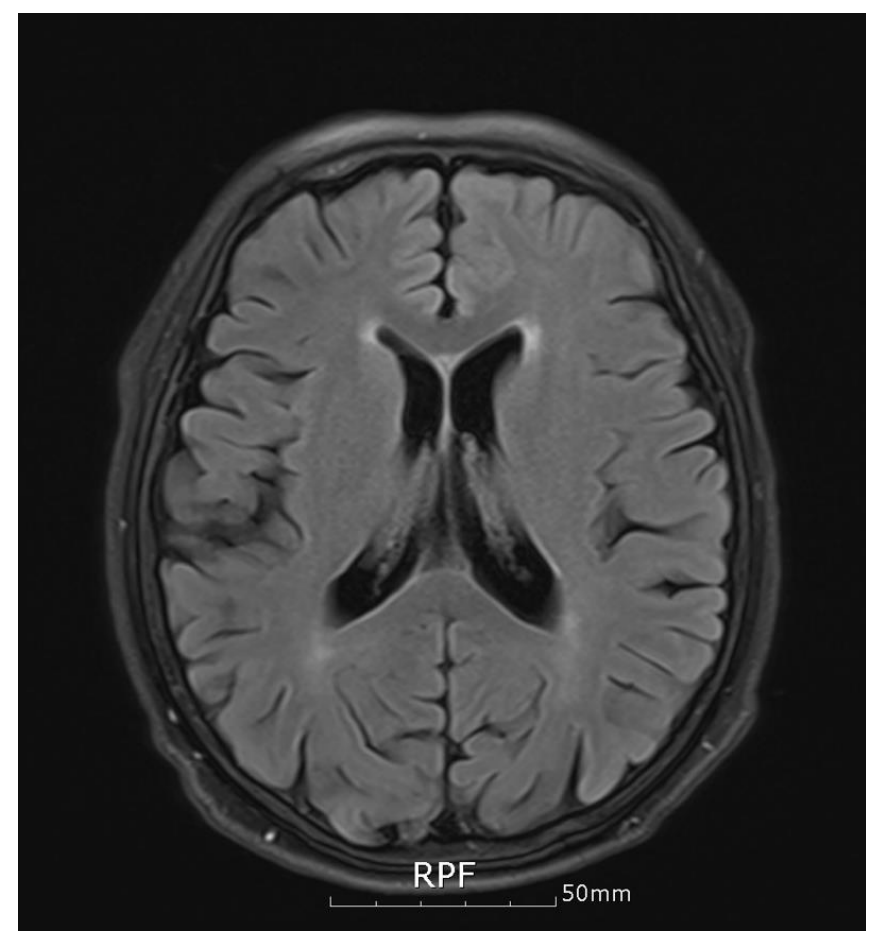

Fig. 1. No degenerative changes on T2-flair image. RPF, right posterior feet.

charge were: methylphenidate $25 \mathrm{mg}$, modafinil $200 \mathrm{mg}$, olanzapine $10 \mathrm{mg}$, donepezil $10 \mathrm{mg}$, and aripiprazole $5 \mathrm{mg}$. As her activity levels increased a little, her daily life function improved slightly but her subjective cognitive decline continued. We will keep track of the changes in her cognitive function in the future.

\section{Discussion}

Apathy is a common behavioral problem that often goes undiagnosed and untreated [4]. There is considerable overlap in the clinical presentations of apathy and depression. Furthermore, apathy may be part of a cluster of negative symptoms in patients with illnesses such as schizophrenia or Alzheimer's disease [5]. However, apathy has been demonstrated to relate, independently of these other psychiatric symptoms, to a variety of outcomes [5]. The presence of apathy is linked to deficits in the performance of activities of daily living and functional decline and can be of considerable clinical significance [4].

A common feature of many of the conditions in which apathy is prominent is the presence of lesions or other abnormalities in the frontal lobe-subcortical circuitry. Neuroimaging studies of various clinical populations have reported correlations between apathy and structural and functional changes within the frontal lobe, particularly the anterior cingulate gyrus and subregions of the basal 

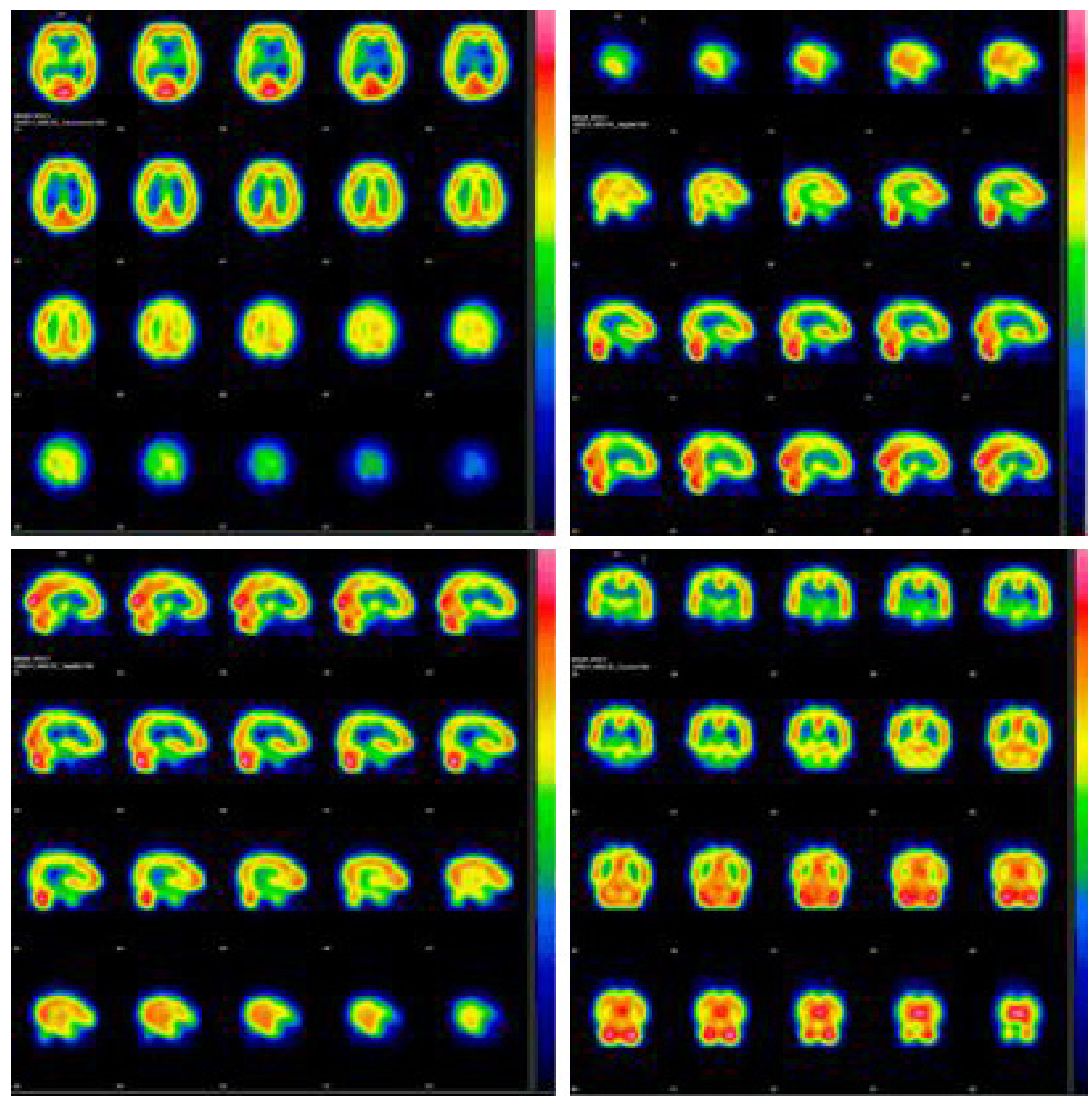

Fig. 2. Mild reduction of perfusion in anterior cingulate gyrus on brain single-photon emission computed tomography.

ganglia. Neuropsychological studies have reported a relationship between apathy and poorer performance on tests of executive functions [6]. Indeed, in the present case, the patient displayed decreased function of the anterior cingulate gyri as well as deterioration of executive functions.

A recent case-control study [7] reported that apathy appeared to be greater in patients who were treated with SSRI than in pa- tients who were not. Currently, we do not have enough data to understand how altering of serotonergic signaling might cause apathy. It is biologically possible that frontal lobe dysfunction, induced by SSRIs, may be responsible for the apathy [7]. Conditions that can induce frontal lobe dysfunctions are often caused by imbalances of neurotransmitters in the brain. Prolonged and excessive serotonin in the synapse may lead to a decrease in the 
transmission of dopamine in the frontal lobe. High serotonin levels may also cause a decrease in acetylcholine which can induce an increase in dopamine function. A relationship between serotonin and noradrenaline is another possible mechanism [8].

If SSRI-induced apathy is detected, reduction of SSRI dose or discontinuation of SSRIs is required. When discontinuation of the SSRI is not successful, augmentation or switching to a different class of medications is helpful [2]. Apathy has been emerging as a target for pharmacotherapeutic interventions, and new trials have recently been completed [9]. Methylphenidate for the treatment of apathy has been investigated in double-blind, placebo-controlled randomized trials (RCTs), and an effect on apathy was reported [10]. The findings of these studies were consistent with other findings supporting dopaminergic dysfunction in apathy. Other catecholamine agonists that can treat the symptoms of apathy include amantadine [11], bromocriptine [12], levodopa [13], selegiline [14], and bupropion [15]. RCTs have provided evidence of the efficacy of cholinesterase inhibitors [16] and memantine [17] in the treatment of apathy in Alzheimer's disease. Modafinil is a multimodal stimulant that increases the activity of histamine, norepinephrine, serotonin, dopamine, and orexin systems in the brain [18]. Two recent case reports [4,19] suggested its effectiveness in the treatment of apathy. Additionally, several studies have investigated the effects of olanzapine on negative symptoms, including apathy. Apathetic patients with major depressive disorder may benefit from treatment with olanzapine. In an open-label study [20] with nonpsychotic, major depressive disorder patients who were treated with SSRIs and displayed significant apathy, the addition of olanzapine in the treatment regime yielded a significant decrease on the AES.

In the present case, the apathy symptoms improved slightly but the patient continued to experience cognitive impairment. Apathy is the most common behavioral problem associated with Alzheimer's dementia, affecting $70-90 \%$ of patients, and the presence of apathy is linked to deficits in the performance of activities of daily living and functional decline [4]. In one study, minor cognitive impairment (MCI) patients with apathy symptoms, not depressive symptoms, were diagnosed with Alzheimer's dementia almost seven times more frequently than MCI patients without apathy symptoms. Patients with apathy should keep track of their cognitive function, taking into consideration the risk of dementia.

The growing body of empirical investigations on the neurobiology of apathy will likely prove helpful in providing a sound theoretical basis for the application of currently available treatments, as well as for the development of novel therapeutic interventions [5].

\section{Conflicts of interest}

No potential conflicts of interest relevant to this article was reported.

\section{ORCID}

Hye-Guem Kim, https://orcid.org/0000-0002-9677-7011

Bon-Hoon Koo, https://orcid.org/0000-0001-9633-3835

Seung Woo Lee, https://orcid.org/0000-0002-3730-066X

Eun-Jin Cheon, https://orcid.org/0000-0002-6986-0768

\section{References}

1. Marin RS. Apathy: a neuropsychiatric syndrome. J Neuropsychiatry Clin Neurosci 1991;3:243-54.

2. Barnhart WJ, Makela EH, Latocha MJ. SSRI-induced apathy syndrome: a clinical review. J Psychiatr Pract 2004;10:196-9.

3. Starkstein SE, Jorge R, Mizrahi R, Robinson RG. A prospective longitudinal study of apathy in Alzheimer's disease. J Neurol Neurosurg Psychiatry 2006;77:8-11.

4. Padala PR, Burke WJ, Bhatia SC. Modafinil therapy for apathy in an elderly patient. Ann Pharmacother 2007;41:346-9.

5. Roth RM, Flashman LA, McAllister TW. Apathy and its treatment. Curr Treat Options Neurol 2007;9:363-70.

6. McPherson S, Fairbanks L, Tiken S, Cummings JL, Back-Madruga C. Apathy and executive function in Alzheimer's disease. J Int Neuropsychol Soc 2002;8:373-81.

7. Wongpakaran N, van Reekum R, Wongpakaran T, Clarke D. Selective serotonin reuptake inhibitor use associates with apathy among depressed elderly: a case-control study. Ann Gen Psychiatry 2007;6:7.

8. Dubini A, Bosc M, Polin V. Do noradrenaline and serotonin differentially affect social motivation and behaviour? Eur Neuropsychopharmacol 1997;7(Suppl 1): S49-55.

9. Lanctôt KL, Agüera-Ortiz L, Brodaty H, Francis PT, Geda YE, Ismail Z, et al. Apathy associated with neurocognitive disorders: Recent progress and future directions. Alzheimers Dement 2017;13:84-100.

10. Galynker I, Ieronimo C, Miner C, Rosenblum J, Vilkas N, Rosenthal R. Methylphenidate treatment of negative symptoms in patients with dementia. J Neuropsychiatry Clin Neurosci 1997;9:231-9.

11. van Reekum R, Bayley M, Garner S, Burke IM, Fawcett S, Hart A, et al. $\mathrm{N}$ of 1 study: amantadine for the amotivational syndrome in a patient with traumatic brain injury. Brain Inj 1995;9:49-53.

12. Marin RS, Fogel BS, Hawkins J, Duffy J, Krupp B. Apathy: a treat- 
able syndrome. J Neuropsychiatry Clin Neurosci 1995;7:23-30.

13. Debette S, Kozlowski O, Steinling M, Rousseaux M. Levodopa and bromocriptine in hypoxic brain injury. J Neurol 2002; 249:1678-82.

14. Newburn G, Newburn D. Selegiline in the management of apathy following traumatic brain injury. Brain Inj 2005; 19:149-54.

15. Corcoran C, Wong ML, O'Keane V. Bupropion in the management of apathy. J Psychopharmacol 2004;18:133-5.

16. Holmes C, Wilkinson D, Dean C, Vethanayagam S, Olivieri S, Langley A, et al. The efficacy of donepezil in the treatment of neuropsychiatric symptoms in Alzheimer disease. Neurology 2004;63:214-9.

17. Zhang N, Wei C, Du H, Shi FD, Cheng Y. The effect of meman- tine on cognitive function and behavioral and psychological symptoms in mild-to-moderate Alzheimer's disease patients. Dement Geriatr Cogn Disord 2015;40:85-93.

18. Gerrard P, Malcolm R. Mechanisms of modafinil: a review of current research. Neuropsychiatr Dis Treat 2007;3:349-64.

19. Camargos EF, Quintas JL. Apathy syndrome treated successfully with modafinil. BMJ Case Rep 2011;2011:pii: bcr0820114652.

20. Marangell LB, Johnson CR, Kertz B, Zboyan HA, Martinez JM. Olanzapine in the treatment of apathy in previously depressed participants maintained with selective serotonin reuptake inhibitors: an open-label, flexible-dose study. J Clin Psychiatry 2002;63:391-5. 